\title{
Los microempresarios del altiplano y el capital semilla, una visión desde la antropologia empresarial
}

\section{The micro-entrepreneurs of the highlands and the seed capital, a vision from the entrepreneurial anthropology}

Tomás Véliz Quispe ${ }^{1, \mathrm{a}} \underline{0000-0003-4383-0365}$

Carol Fabiola Véliz Gonzales ${ }^{2, b} \underline{0000-0002-9078-0762}$

${ }^{1}$ Universidad Nacional del Altiplano, Puno, Perú.

${ }^{2}$ Universidad Católica San Pablo, Arequipa, Perú.

atveliz@unap.edu.pe

bcarol.veliz.gonzales@ucsp.edu.pe
Recibido el : 21/06/2021

Aceptado el : 22/10/2021

Publicado el:15/12/2021

\section{Resumen}

La investigación destaca las bases e importancia del capital para los microempresarios del altiplano del Perú al 2020; en un contexto de globalización del capital. El objetivo es describir e interpretar la naturaleza del "capital semilla y cultural" de los microempresarios andinos, desde la perspectiva de la antropología empresarial. La metodología está enmarcada en el paradigma cuantitativo y cualitativo; diseño no experimental y tipo descriptivo; muestra 26 encuestas y 11 testimonios de microempresarios; técnica de encuesta por cuestionario y entrevista. El estudio se realizó en los meses de febrero 2018 a marzo del 2020. Los resultados: El capital de la microempresa del altiplano destaca: 1. Capital semilla (ahorros de trabajo, prestamos familiares, uso de crédito) 2. Capital cultural (valores de reciprocidad, responsabilidad y respeto) 3. Capital simbólico (creencias y rituales el tributo a la tierra con vino o cerveza; símbolos de prosperidad la ruda, flores amarillas y lentejas). Las conclusiones: El enfoque del capital desde la antropología empresarial en el altiplano considera: dimensión económicasemilla, cultural-valores y simbología- rituales forman parte de la filosofía de la microempresa.

Palabras clave: Capital cultural; capital económico; capital semilla; capital simbólico; capital social; microempresario.

\begin{abstract}
The research highlights the basis and importance of capital for microentrepreneurs in the Peruvian highlands in 2020; in a context of globalization of capital. The objective is to describe and interpret the nature of "seed and cultural capital" of Andean microentrepreneurs, from the perspective of business anthropology. The methodology is framed in the quantitative and qualitative paradigm; nonexperimental and descriptive design; sample 26 surveys and 11 testimonies of microentrepreneurs; questionnaire survey and interview technique. The study was conducted in the months of February 2018 to March 2020. The results: The capital of the microenterprises in the highlands stands out: 1. Seed capital (work savings, family loans, use of credit) 2. Cultural capital (values of reciprocity, responsibility and respect) 3. Symbolic capital (beliefs and rituals: tribute to the earth with wine or beer; symbols of prosperity: rue, yellow flowers and lentils). The conclusions: The capital approach from the entrepreneurial anthropology in the highlands considers: economic dimension-seed, cultural-values and symbolism-rituals are part of the philosophy of the microenterprise.
\end{abstract}

Keywords: Cultural capital; economic capital; seed capital; symbolic capital; social capital; microentrepreneur. 


\section{Introducción}

El capital es la base de la prosperidad económica en el sistema capitalista y va adquiriendo importancia en los países emergentes. Alude De Soto (2004) "El capital es la sabia del sistema capitalista, el cimiento del progreso e irónicamente es justo aquello que países pobres del mundo parecen no poder producir, no importa con cuanto afán su gente practique todas las demás actividades que definen a una economía capitalista” (p. 91).

Los microempresarios frente a la globalización del capital y mercados, han adaptado flexiblemente las necesidades del capital y la gestión de microempresas, se destacan iniciativas que promueven la microempresa al interior del Perú. Para Tahmasebi y Askaribezayeh (2021) las iniciativas de microfinanzas promueven considerablemente el capital social de la comunidad y la participación en las actividades de desarrollo rural. También destaca De Althaus (2009) la revolución capitalista en el Perú y el capital ha empezado a civilizar regiones y áreas de la economía y articular mejor la estructura productiva al interior del país.

Sobre "Capital semilla" o capital inicial hace referencia Echecopar (2006) en la etapa de gestación del emprendimiento las principales fuentes de financiamiento son los ahorros del emprendedor y el apoyo de familiares y amigos. El primer capital de contribución se destaca actualmente en muchos microempresarios para fortalecer su sostenibilidad económica.

En su perspectiva integradora Reyes et al. (2018) relacionan la formación del capital en económico, cultural y social en el emprendimiento empresarial. Se suma con una asociación y relación del capital Korkeila y Hamari (2020) el económico con orientación hacia el logro y el lado de relación, el social con orientación a la inmersión y las partes sociales e intereses, el simbólico con la orientación al logro y permanencia social. Se destaca en las microempresas locales la integración del capital semilla y cultural muy significados para él éxito y sostenibilidad de sus negocios.

La contribución de la investigación se centra en la profundización del estudio del capital de los microempresarios, en una perspectiva integradora e inclusiva con enfoque de la antropología económica en un contexto de globalización empresarial. La presión de la globalización acelera la reinvención de la cultura hacia un modelo de desarrollo económico (Salas et al., 2019).

El objetivo de la investigación es: Describir e interpretar la naturaleza del "capital semilla y cultural" de los microempresarios del altiplano, desde la perspectiva de la antropología empresarial.

\section{Marco teórico}

\section{Los microempresarios y sus actividades económicas}

Los microempresarios en la economía rural y urbana, lo destacan Malengreau (1974) "Los empresarios comerciantes venden los bienes obtenidos de ganadería comercial, en el pueblo o mercado regional, y sirven de intermediarios para la venta en el pueblo de productos de una economía agrícola asalariada y de consumo de la industria nacional o extranjera" (p. 178) y Espinoza (1998) en las ciudades se concentra en: Calzado y prendas de vestir, muebles, panadería y derivados, manufactura, maquinaria y equipos, cuero, madera $\mathrm{y}$ productos químicos; abarrotes, ferretería, restaurantes, pollerías, artesanía, transporte, etc.

Los microempresarios en la región comercializan en ferias rurales productos agropecuarios, granos andinos $\mathrm{y}$ en centros comerciales publicitan marcas globales y nacionales demandas por la clase media emergente (Coca-cola, Bimbo, Gloria, Nike, Adidas, Puma, Calvin Klein, etc.).

\section{La integración del capital semilla y cultural desde una perspectiva de la antropología empresarial}

Se esbozan dos iniciativas para la definición del capital semilla. Según Umaña (2019); Barona y Gómez (2010) el capital semilla o inicial da comienzo el primer año a un negocio, nace de activos personales, ahorros, familia y amigos; siguen en su orden el crédito empresarial.

Señala Loayza (2006) "Puede afirmarse además del capital económico, el empresario hace uso de un capital social, definido como el conjunto de redes sociales a las cuales recurre para salir adelante y plasmar su proyecto empresarial, así como utilizar mejor los recursos con los que cuenta incluyendo el uso del crédito" (p. 121). En opinión de Llanque (2011) La reciprocidad 
familiar y social contribuyen a formar el capital económico, incluye paisanos y qamiris (ricos), en fiesta matrimonial otorgan importante contribución económica y social, para que inicien sus actividades. Para Gonzales et al. (2021) el fortalecimiento del capital social es producto de la interacción del grupo y se complementa con préstamos, generando ventaja competitiva para rentabilidad de la empresa. Entonces subyacen para formar el capital económico y emprendimientos apoyo de redes parentales, familiares y préstamos de autofinanciamiento.

En otro contexto del capital cultural para Grzegorczyk(2019) lasculturasnacionalesasimilan la mirada del capital social y revelan rasgos de la realidad que de otro modo permanecen invisibles, estos juegan un papel clave en el funcionamiento de todas las economías. En opinión de Claverías (s/f) empresarios exitosos en la costa urbana en Perú, son inmigrantes y originarios de las culturas andinas y amazónicas; se destaca factores para el éxito: El capital cultural, capital social y capital humano (entendido como capital conocimiento). Para los microempresarios el capital cultural son sus valores de herencia cultural y de cosmovisión andina y persisten en prácticas empresariales, normas de comportamiento en la microempresa.

El capital social, señala Ávalo et al. (2016) cuenta con sub-variables de confianza, apoyo, interés relacional e interdependencia y estos son articuladores de la acción y conocimiento. En la cultura aymara Santos (2007); Llanque (2011) señalan parte de la identidad, articulada a preservar y conservar la cultura, tradiciones y la biodiversidad; la formación de un rico en cuanto a su ética de trabajo y valores la responsabilidad, tenacidad, respeto mutuo, reciprocidad, compromiso social, religiosidad y prácticas creyentes en los negocios. Sintetiza en términos generales los valores andinos de colaboración y colectivos Golte (1990) Asistencia mutua, trabajo comunal, ahínco, sobriedad, frugalidad y ética laboral.

En otra posición establece Padilla y Ciruela (2018) los valores culturales de colaboración (ayni) no están siendo aplicados en el emprendimiento, en sus contactos sociales relacionados con el negocio, siendo el individualismo más común.

El capital simbólico identifica la participación en creencias de sincretismo religioso del microempresario andino. Según Arias et al. (2013); Cajías (2009); Coraza (2017) en los rituales de la última cosecha y primera siembra, en fiestas patronales en honor a: Deidades del panteón católico (Dios, Cristo, la virgen, santísima cruz, los santos etc.) y deidades del panteón andino (La pachamama, apus y achachilas (espíritus tutelares) tienen confianza en la protección que les brindan y agradecen con ritos en su honor.

Precisa Barreto (2007) la tradición está vigente y puede ser fuente de prosperidad económica y reafirmación simbólica. Considera Mayta (2019); Los Andes (04 de junio 2019); Arrosquipa (2014) la práctica de rituales es recreada por actores de la micro y pequeña empresa e inciden en la gestión y planificación; la ofrenda de la "dulce misa", anhelo de suerte, éxito; e imágenes de negocios y trabajo (tiendas de abarrotes, ferreterías, farmacias etc.); la costumbre de limpia de casas y negocios con humo de incienso, echan mixturilla amarilla, chicha y hojas de coca alrededor; baldear la acera con ruda para atraer ventas, jalador de clientes; y se extiende también en el proceso de comercialización de la fibra. Los rituales andinos los clasifica Arnold (2004) en: Ritos domésticos, laborales, comerciales, políticos y religiosos.

Señalan Golte y Adams (1990); Llanque (2011) el capital económico, cultural, social y simbólico que migrantes traen consigo, son influidos por experiencias en sus comunidades de origen como en las de destino. Ya involucrados en el proceso migratorio, aportan esos capitales de herencia para la creación de contextos en lugares diferentes basados en su significado cultural.

\section{Materiales y métodos}

En tanto la naturaleza epistemológica del estudio es conocer el "Capital semilla y cultural" y su relevancia en la práctica por los microempresarios, desde el enfoque de la antropología empresarial frente a la globalización del capital.

La investigación se enmarca en el objeto de estudio capital semilla y cultural, su contribución en la lógica de los microempresarios. Utiliza la metodología mixta de investigación cualitativa y cuantitativa. Según Johnson \& Onwuegbuzie (2004) es un tipo de investigación y se combinan técnicas, métodos, aproximaciones, conceptos o lenguaje cualitativo y cuantitativo dentro de una misma investigación; en una lógica de 
aproximación constructivista y pragmatista de la investigación.

Este diseño de investigación mixto, va más allá de la triangulación para ver convergencias o divergencias de resultados; y como iniciación para descubrir nuevas perspectivas, complementariedad de diferentes facetas, expansión, combinación y ampliación de focalización y desarrollo secuencial. El procedimiento del estudio se ha organizado por etapas: 1. Preguntas de investigación 2. Recolección de datos 3. Análisis de datos. 4. Interpretación de la información 5. Triangulación y 6. Conclusiones.

Seutilizómetodologíay técnica activa, construcción de testimonios y normas de comportamiento. La unidad de análisis consideró una muestra de 11 microempresarios informantes.

El estudio se realizó en los meses de febrero 2018 a marzo del 2020. El ámbito de georreferencia de la investigación se desarrolla en las microempresas de la región del Altiplano del Perú. Según Arias et al. (2013) en la región se identifican zonas etnolingüísticas: Aymara norte y sur (español/ aymara), quechua (español/quechua) y mixtas influenciadas por centros urbanos mestizos (español/quechua/aymara).

Se muestra en la tabla $\mathrm{N}^{\circ} 1$, informantes considerando su zona de origen y desarrollo de actividades de comercio, producción y servicios.

250 Tabla 1. Microempresarios informantes según zona de origen.

\begin{tabular}{|c|c|c|}
\hline Actores & Zona de origen & $\begin{array}{l}\text { Actividades } \\
\text { económicas }\end{array}$ \\
\hline Microempresarios & $\begin{array}{l}\text { Juli (02), Ilave (01), } \\
\text { Azángaro (01), } \\
\text { Melgar (02), Lampa } \\
(01) \text {, Juliaca (02); } \\
\text { Puno (02) }\end{array}$ & $\begin{array}{l}\text { - De comercio, } \\
\text { producción y } \\
\text { servicios }\end{array}$ \\
\hline Total & 11 & \\
\hline
\end{tabular}

La recolección de datos se llevó a cabo con entrevista abierta, los testimonios de informantes clave han sido determinantes y se transcribió para su análisis.

En la unidad de análisis cuantitativa, estuvo representada una muestra de 26 microempresarios en diferentes rubros económicos, el muestreo intencionado consideró la formalización legal y buenas prácticas de gestión. En la tabla $\mathrm{N}^{\circ} 2$, se muestra actores según actividades económicas.

Tabla 2. Microempresarios informantes actores de negocios.

\begin{tabular}{lll}
\hline Actores & $\begin{array}{l}\text { Zona de } \\
\text { origen }\end{array}$ & Actividades económicas \\
\hline Microempresarios & $\begin{array}{l}\text { - Aymara } \\
\text { - Quechua }\end{array}$ & $\begin{array}{l}\text { - Emprendimientos de } \\
\text { venta de ganado, } \\
\text { transporte interprovincial, } \\
\text { truchícolas, producción de } \\
\text { lácteos, confecciones, } \\
\text { turismo rural, tiendas } \\
\text { bodegas. }\end{array}$ \\
& & \\
\hline Total & $\mathbf{2 6}$ & \\
\hline
\end{tabular}

Fuente. Entrevistados microempresarios, región Puno, 2018 - 2020.

En la recolección de datos se aplicó un cuestionario estructurado a los actores encuestados:

¿Cómo obtuvo su "Capital semilla" para el negocio?

¿Qué valores se practican en su negocio?

¿Qué ceremonias (ritos) y creencias se practican para la prosperidad de su negocio?

La técnica de análisis de datos y medición fue con estadística descriptiva.

\section{Resultados}

Análisis cualitativo

Categoría: Capital económico.

El dinero es considerado para el progreso económico de las familias y se destaca en el espíritu emprendedor de microempresarios. Sobre el tema nos cuenta John Tapia Microempresario de Juli:

Mi primer capital fueron mis ahorros cuando trabajaba de estudiante, ahora si hay eso de casarse por conveniencia y eso es el ayni fiesta, que se celebra en la zona aymara fiesta que debe visitar con un cuasi monto económico, eso se llama regalo y se convierte en un compromiso a futuro que se debe devolver.

Los microempresarios valoran la sociabilidad y amistad de parentesco, las fiestas constituyen lugares de acercamiento a su vida futura, es costumbres sumas de dinero, artefactos, cerveza y hasta se guarda parte de los obsequios y después 
son devueltos a las empresas y se convierten en su capital inicial.

\section{Categoría: Capital cultural.}

Es característico en microempresarios los valores andinos y se trasmitan u orientan al progreso social, iniciativa y riesgos que supone el desarrollo de la microempresa. Al respecto nos manifiestan los microempresarios Leonidas Incacutipa, Roberto Apaza y Martín Canahua:

Nosotros practicamos el apoyo a sí mismo no como el individualismo moderno, si una empresa necesita apoyo de mano de obra le dice que la va a apoyar y cuando necesitas me apoyas, manejamos con transparencia nuestros repartos, cuando no hay productos para vender en nuestras zonas salimos a buscar. Estamos pendientes de las ferias, existe una ayuda mutua compartida, cumplimos con deberes y compromisos que se tiene, somos voluntariosos con lo que hacemos, respetuosos de nuestras creencias y semejantes y nos consideramos decentes, decorosos y justos. Por mi zona abundan empresas truchícolas, a pesar que casi la mitad de truchas mueren en la producción; nos prestamos entre nosotros para cumplir nuestros compromisos, somos responsables y respetamos la lealtad entre nosotros para buscar el progreso.

Los microempresarios en sus prácticas y normas de comportamiento guardan valores de su cultura, estos guían su actuar en el mercado; la perseverancia y respeto, ayuda mutua de reciprocidad, lealtad entre ellos, preservan la relación con clientes.

\section{Categoría: Capital simbólico}

Las ceremonias y práctica de ritos y creencias en microempresarios es parte de la naturaleza en los negocios. Al respecto nos cuentan los microempresarios Nancy Turpo, Francisco Mamani, María Camapaza, Adrián Ramírez, Elsa Quenta, María Choquehuayta y Juan Choque:

Al inicio de junio en la fiesta de Trinidad, se arman fogatas y hace "challado" se agradece a la santa tierra, apus y ganado para que prospere la reproducción, en agosto se hace pago a la tierra y se tributa honor a la piedra con el saumeo (Humeado) para tener dinero; al iniciar el mes caminamos temprano al cerro Huayna Roque, hacemos oración católica en círculo, se lleva vino, coca y en una lliclla (manto) se hace el ritual de la coca, conversamos sobre metas y reducción de mora, en los salones el saumeo al dinero, fe para que crezca y puedan pagar; en el ramo textil confecciones, cuando abrimos una nueva empresa la maquinaria textil se "challa", flores para empezar con buen pie y éxito; en lácteos realizamos tributo a la santa tierra, con nuestros colaboradores, un compartir de karmu (comida), ideales de mejora, consejos, bromas etc.; en turismo vivencial, al inicio de mes, realizamos pago a la tierra, usamos traje del lugar para que nos vaya bien, lo mismo hacemos con un nuevo trabajador; por la mañana empezamos con la limpieza, echamos agua de ruda del día anterior; lenteja para la buena suerte y nunca falte dinero.

Ciertamente el microempresario se apoya en rituales ceremoniales y creencias populares que le provocan sus bienestar y éxito en sus negocios; en provincias es más notorio el tributo de agradecimiento a la naturaleza bajo un sincretismo religioso; en las capitales de ciudad destaca la challa en agradecimiento a la santa tierra, creencias como las flores, la ruda y lenteja, están arraigadas creencias para prosperidad de la microempresa.

Análisis cuantitativo:

Tabla 3. Capital económico: Origen del capital semilla en la microempresa.

\begin{tabular}{lll}
\hline $\begin{array}{l}\text { Fuentes de capital semilla para los } \\
\text { microempresarios }\end{array}$ & Fr. & \% \\
\hline Préstamos de entidades. & 5 & 19 \\
Ahorros de trabajo dependiente. & 12 & 46 \\
Prestamos solidario familiar. & 4 & 16 \\
Ahorros de trabajo independiente. & 4 & 16 \\
Otros & 1 & 3 \\
\hline Total & $\mathbf{2 6}$ & $\mathbf{1 0 0}$ \\
\hline Fuente. Muestra el capital semilla con encuesta a microempresarios, Puno, \\
2020.
\end{tabular}

En la Tabla $\mathrm{N}^{\circ}$ 3, se muestra el origen de las fuentes del capital económico con el que empiezan los microempresarios, el $46 \%$ ahorros de trabajos dependientes, el $19 \%$ prestamos de entidades financieras, el $16 \%$ de préstamos de la familia, el $16 \%$ de ahorros de trabajo independiente y $3 \%$ de préstamos informales. 
Tabla 4. Capital cultural: Valores relevantes en la práctica de la microempresa.

\begin{tabular}{lll}
\hline Práctica de valores en la microempresa & Fr. & $\mathbf{\%}$ \\
\hline Apoyo a si mismo & 6 & 23 \\
Transparencia & 2 & 8 \\
Respeto & 4 & 15 \\
Justicia & 2 & 8 \\
Responsabilidad & 6 & 23 \\
Lealtad & 4 & 15 \\
Otros & 2 & 8 \\
\hline Total & $\mathbf{2 6}$ & $\mathbf{1 0 0}$ \\
\hline
\end{tabular}

Fuente. Muestra el capital cultural con encuestas a microempresarios, Puno, 2020.

En la tabla $\mathrm{N}^{\circ} 4$, se muestra la práctica de valores en la microempresa, el $23 \%$ la reciprocidad entre ellos, el 23\% la responsabilidad, el 15\% el respeto, el 15\% la lealtad, el $8 \%$ la transparencia, el $8 \%$ la justicia y el $8 \%$ otros valores como la perseverancia, la innovación, la honestidad y puntualidad.

En la tabla $\mathrm{N}^{\circ} 5$, se muestra la práctica de ritos y creencias para prosperidad de microempresas; el $35 \%$ el tributo a la tierra, el $19 \%$ creencia en el agua de ruda, el $11 \%$ la lenteja, un $15 \%$ otras creencias las flores, mixturilla etc.
Tabla 5. Capital simbólico: Ceremonias (ritos) y creencias en la microempresa.

\begin{tabular}{lll}
\hline $\begin{array}{l}\text { Práctica de Ceremonias (ritos) y creencias } \\
\text { para prosperidad de la microempresa }\end{array}$ & Fr. & $\mathbf{\%}$ \\
\hline Tributo a la tierra (challachi) & 9 & 35 \\
Ofrendas a los cerros & 2 & 8 \\
Humeado a la piedra & 1 & 4 \\
Saumeo al dinero & 2 & 8 \\
Agua de ruda & 5 & 19 \\
Lenteja & 3 & 11 \\
Otros & 4 & 15 \\
\hline Total & $\mathbf{2 6}$ & $\mathbf{1 0 0}$ \\
\hline Fuente. Muestra el capital simbólico con encuestas a microempresarios, \\
Puno, 2020.
\end{tabular}

\section{Discusión}

Triangulación de datos cualitativos y cuantitativos "Capital semilla y cultural" en microempresas.

En este apartado se detalla las tendencias producto de la triangulación de datos para las distintas categorías del capital semilla y cultural en las microempresas.

Categoría: Capital económico.

Tabla 6. Capital económico: Origen y relevancia en la microempresa.

\begin{tabular}{|c|c|c|c|c|}
\hline $\begin{array}{l}\text { Variable / } \\
\text { Dimensión }\end{array}$ & $\begin{array}{l}\text { Entrevista } \\
\text { Cualitativa }\end{array}$ & $\begin{array}{l}\text { Cuestionario } \\
\text { Cuantitativo) }\end{array}$ & $\begin{array}{l}\text { Revisión de } \\
\text { literatura }\end{array}$ & $\begin{array}{l}\text { Concordancia } \\
\text { y/o diferencias }\end{array}$ \\
\hline $\begin{array}{l}\text { Capital } \\
\text { económico. }\end{array}$ & $\begin{array}{l}\text { Mi primer capital } \\
\text { fueron mis ahorros } \\
\text { cuando trabajaba de } \\
\text { estudiante. Ahora hay } \\
\text { eso de casarse por } \\
\text { conveniencia (ayni } \\
\text { fiesta), visitar con un } \\
\text { cuasi monto económico } \\
\text { o regalo y eso se } \\
\text { convierte en } \\
\text { compromiso a futuro a } \\
\text { devolver. (Jhon T., } \\
\text { 2019). }\end{array}$ & $\begin{array}{l}46 \% \text { de ahorros } \\
\text { acumulados de } \\
\text { trabajo } \\
\text { dependiente; } 16 \% \\
\text { de préstamos } \\
\text { solidarios } \\
\text { familiares; } 19 \% \\
\text { de préstamos de } \\
\text { entidades } \\
\text { financieras. } \\
\text { (Encuesta - P1) }\end{array}$ & $\begin{array}{l}\text { En sus inicios } \\
\text { destacan ahorros y } \\
\text { apoyo de familiares, } \\
\text { amigos. } \\
\text { (Echecopar, G.: } \\
\text { 2006) } \\
\text { En empresas } \\
\text { caleñas destacan } \\
\text { ahorros de } \\
\text { familiares, amigos y } \\
\text { dueños; sigue el } \\
\text { crédito empresarial } \\
\text { (Barona, B. \& } \\
\text { Gómez, A.; 2010). }\end{array}$ & $\begin{array}{l}\text { Con } \\
\text { concordancia } \\
\text { plena entre los } \\
\text { datos aportados } \\
\text { por las } 3 \text { fuentes } \\
\text { de información. }\end{array}$ \\
\hline
\end{tabular}

Fuente. Muestra el capital económico con contraste de datos cualitativos, cuantitativos y revisión de literatura, Puno, 2020.

En la tabla 6, se muestra el capital semilla, hay una concordancia plena entre los datos de las tres fuentes de información, donde el ahorro producto del trabajo dependiente es impulsor inicial para las microempresas; se ratifica con el $46 \%$ señalan que acumulan ahorros del trabajo, se suma los préstamos de familiares en los inicios de microempresas y concordante aún más con la literatura citada.

El origen del capital semilla con el que inician los microempresarios tiene características económicosociales y está vinculado al financiamiento con ahorros de trabajos dependientes, apoyo de 
familiares y amigos, tal como es coincidente con lo expresado por (Echecopar, 2006). La reciprocidad familiar, se basa en la solidaridad basada en redes familiares de parentesco y amistad cultural; los regalos y colectivos por la apreciación al vínculo matrimonial es respetado en los círculos sociales (Llanque, 2011). El capital económico se integra con el capital social en los inicios del emprendimiento, formándose redes familiares en una misma actividad económica principalmente.

Categoría: Capital cultural.

Tabla 7. Capital cultural: Origen y relevancia en la microempresa.

\begin{tabular}{|c|c|c|c|c|}
\hline $\begin{array}{l}\text { Variable / } \\
\text { Dimensión } \\
\end{array}$ & $\begin{array}{l}\text { Entrevista } \\
\text { Cualitativa }\end{array}$ & $\begin{array}{l}\text { Cuestionario } \\
\text { Cuantitativo) }\end{array}$ & $\begin{array}{l}\text { Revisión de } \\
\text { literatura }\end{array}$ & $\begin{array}{l}\text { Concordancia } \\
\text { y/o diferencias }\end{array}$ \\
\hline $\begin{array}{l}\text { Capital } \\
\text { cultural. }\end{array}$ & $\begin{array}{l}\text { Practicamos el apoyo a sí } \\
\text { mismo; transparencia, } \\
\text { ayuda mutua, cumplimiento } \\
\text { de compromisos, voluntad, } \\
\text { respeto, decencia, decoro, } \\
\text { justicia; responsabilidad y } \\
\text { lealtad. (Leonidas I.; } \\
\text { Roberto A. y Martín C., } \\
\text { 2018). }\end{array}$ & $\begin{array}{l}\text { El 23\% la } \\
\text { reciprocidad; el } \\
23 \% \text { la } \\
\text { responsabilidad; } \\
\text { El 15\% el } \\
\text { respeto, lealtad; } \\
\text { el } 8 \% \text { la } \\
\text { transparencia y } \\
\text { lealtad. } \\
\text { (Encuesta - P2) }\end{array}$ & $\begin{array}{l}\text { En la racionalidad } \\
\text { andina se identifican } \\
\text { valores de asistencia } \\
\text { mutua, trabajo } \\
\text { comunal, ahínco, } \\
\text { sobriedad, y } \\
\text { frugalidad. (Golte, } \\
\text { 1990) }\end{array}$ & $\begin{array}{l}\text { Con } \\
\text { concordancia } \\
\text { moderada entre } \\
\text { los datos } \\
\text { aportados por } \\
\text { las } 3 \text { fuentes de } \\
\text { información. }\end{array}$ \\
\hline
\end{tabular}

En la tabla 7, se muestra el capital cultural, se toma en cuenta los testimonios y encuestas, existe una concordancia moderada respecto al capital cultural, la reciprocidad y responsabilidad son los valores que destacan los microempresarios con el $46 \%$, se ratifica con la literatura considerada.

El capital cultural está basado en la solidaridad (asistencia mutua), respeto personal y dignidad, comunitariedad (trabajo comunal), laboriosidad (ahínco), generosidad, sobriedad como lo sostienen (Llanque, 2011; Golte, 1990). La cultura en esta etapa de negocios es predomínate, en valores de apoyo mutuo, generosidad y respeto a sus vínculos comerciales, estos son principalmente de economía local.

En otra versión diferente Padilla y Ciruela (2018) alude a los valores culturales de colaboración (ayni), estos no están siendo aplicados en sus contactos sociales relacionados con el negocio, siendo el individualismo más común. Es probable que la influencia de globalización del capital tenga influencia en los negocios y en culturas latinoamericanas.

Categoría: Capital simbólico.

Tabla 8. Capital simbólico: Origen y relevancia en la microempresa.

\begin{tabular}{lllll}
\hline $\begin{array}{l}\text { Variable / } \\
\text { Dimensión }\end{array}$ & $\begin{array}{l}\text { Entrevista } \\
\text { Cualitativa }\end{array}$ & $\begin{array}{l}\text { Cuestionario } \\
\text { Cuantitativo) }\end{array}$ & Revisión de literatura & $\begin{array}{l}\text { Concordancia } \\
\text { y/o diferencias }\end{array}$ \\
\hline Capital & Tributo a la tierra, & El 35\% tributo a & En la fiesta como ritual & Con concordancia \\
simbólico. & saumeo al dinero y & la tierra, el 19\% & se integra la simbiosis & plena entre los \\
& piedra; agua de ruda & agua de ruda, 11\% & religiosa católica con la & datos aportados \\
& y lenteja, ofrendas a & lenteja, 8\% & cosmovisión andina & por las 3 fuentes \\
& los cerros. (Nancy & saumeo al dinero & (Cajías, 2009; Coraza, & de información \\
& T., Francisco M.., & (Encuesta - P3) & 2017). Es costumbre & \\
María C., Adrián R., & & echar mixturilla & amarilla, chicha y hojas \\
& Elsa Q., María Ch. y & & de coca; baldear con & \\
& Juan Ch..; (2018 - & & ruda para atraer ventas, & \\
& 2020) & & Mayta, 2019). & \\
& & &
\end{tabular}


En la tabla 8, se muestra el capital simbólico, se toma en cuenta los testimonios y encuestas, existe una concordancia plena respecto al capital simbólico, el tributo a la tierra representa el 35\%, seguido de la creencia en la ruda un $19 \%$, se ratifica con la literatura considerada.

El capital simbólico de los microempresarios de la región es categorizado por Arnold (2004) en: Ritos religiosos, laborales, comerciales, políticos y domésticos. El capital simbólico basado en costumbres, tradiciones, creencias y ritos comerciales para la prosperidad de los negocios; tienen presencia en ceremonias del sincretismo católico y andino, su práctica está vinculada a la cultura y es bastante enraizada y difundida en sus actividades de microempresa.

\section{Conclusiones}

En la microempresa del altiplano, el capital semilla es el ahorro acumulado de trabajos dependientes, préstamos solidarios de familiares y prestamos de entidades de micro finanzas para emprender negocios.

El capital cultural y simbólico destaca valores de reciprocidad sostenido en redes familiares, responsabilidad y respeto; lo simbólico se manifiesta en los rituales y creencias como el tributo a la tierra con vino o cerveza, uso de ruda, flores amarillas y lentejas para la suerte y prosperidad de la microempresa.

Bajo el enfoque de antropología empresarial para la microempresa del altiplano, se propone una matriz del capital: 1. Filosofía de negocios 2. Capital: Económico (semilla), socio-cultural (valores) y simbólico (ritos, creencias y símbolos); esta se complementa con un enfoque de gestión de etno-negocios.

\section{Agradecimientos}

A la Universidad Nacional del Altiplano de Puno por impulsar y el apoyo en la ejecución del estudio a través de FEDU

\section{Referencias bibliográficas}

Arias, A., Rivera, E., Rodríguez, H. y Cano. (2013). Puno en los albores del siglo XXI elementos para educar en la interculturalidad. Puno: Editorial Altiplano EIRL.

Arrosquipa, P. (2014). El lugar de la cultura y la cultura del lugar: prácticas y conocimientos de los criadores de camélidos en el sur peruano. Chungará (Arica), 46(2), 259- 270. 10.4067/ S0717-73562014000200007

Ávalo, JA., Yanque, JL, Cangahuala, G. (2016). El capital social y la planificación adaptativa en la comunidad industrial innovadora del Perú. Estudios gerenciales. 32(139), 162-169.

Barona, B. y Gómez, A. (2010) Aspectos conceptuales y empíricos de la financiación de empresas en Colombia. Cuadernos de Administración (Universidad del Valle), (43), 81-97

Barreto, M. (2007). Turismo cultural, relaciones, contradicciones y expectativas. Colección pasos edita $\mathrm{N}^{\circ} 1$. Tenerife España: Asociación canaria de antropología.

Cajias, F. (noviembre 2009) Relaciones sociales e identidad en fiestas urbano mestizas de la región andina de Bolivia. Lima. En fiestas y rituales Perú: Memorias X encuentro. Instituto nacional de Cultura.

Claverías, R. (s/f) El tesoro de los andes y los nuevos empresarios exitosos. CIED- Perú. Disponible en: https://www.yumpu.com/es/ document/view/10567396/el-tesoro- de-losandes-y-los-nuevos-empresari Claverías osexitosos-en-cied. Recuperado el 4 de enero 2021.

Coraza, W. (1 agosto 2017). Feliz día Pachamama: Que el despertar de la tierra traiga paz, amor y prosperidad. Recuperado el 21 de agosto de 2020. De http://cuzcoeats.com/es/feliz-diapachamama-que-el-despertar-de-la-tierratraiga-paz-amor-y-prosperidad/

De Althaus, J. (2009). La revolución capitalista en el Perú. Perú: Publicaciones el Comercio.

De Soto, H. (2004). El misterio del capital. Bogotá, Colombia: Editorial Planeta Colombiana S.A. 
Echecopar, G.; Angelleli, P.; Galleguillos, G. y Schorr, M. (2006). Capital semilla para el financiamiento de las nuevas empresas. Avances y lecciones aprendidas en América Latina. Washington: Banco Interamericano de Desarrollo. Washington D.C.

Espinoza Herrera, N. (1998). Estrategias de promoción y desarrollo del sector pymes en el perú. Gestión en El Tercer Milenio, 1(1),101110. $10.15381 / \mathrm{gtm} . \mathrm{v} 1 \mathrm{il} .10107$

Gonzales, RG., D'espallier., Merslan, R. (2021) Bifurcación en la rentabilidad empresarial: Una simulación de homofilia basada en agentes en grupos autofinanciados. Revista de investigación empresarial. 129, 495-514. 10.1016/j.jbusres.2020.06.051

Golte, J., \& Adams N. (1990). Los caballos de Troya de los invasores estrategias campesinas en la conquista de la gran Lima. Lima: IEP Ediciones.

Grzegorczyk, M. (2019) El papel del capital social moderado por la cultura en la transferencia de tecnología: Perspectiva de Asia y América. Pronóstico tecnológico y cambio social. 143, 132-141. 10.1016/j.techfore.2019.01.021

Johnson, B. y Onwuegbuzie, A. (2004). Investigación de métodos mixtos: un paradigma de investigación cuyo momento ha llegado. Educational Researcher, 33(7), 1426. 10.3102/0013189X033007014

Korkelia, H. y Hamari, J. (2020). Capital avatar: Las relaciones en la orientación del jugador y el capital social, simbólico, económico y cultural de su avatar. Computadoras en el comportamiento humano. 102, 14-21. $\underline{10.1016 / j . c h b .2019 .07 .036}$

Loayza, J. (2006). Los microempresarios vistos desde la antropología. Año 8, $\mathrm{N}^{\circ} 38$, Arequipa agosto 2006. Publicación de la Universidad Nacional de San Agustín.

Los andes. (04 de junio 2019) Ruda: Poderosa protección contra la energía negativa. Los andes. Recuperado 26 septiembre 2020, de https://www.losandes.com.pe/2019/06/04/ ruda-poderosa-proteccion-contra-la-energianegativa/

Llanque, Jorge. (2011). Qamiris aymaras: Nuevas elites en Oruro. Tinkazos, 14(29), 45-64. Recuperado en 15 de agosto de 2021, de http:// www.scielo.org.bo/scielo.php?script $=$ sci arttext\&pid=S1990-74512011000100004\&ln $\mathrm{g}=\mathrm{es} \& \operatorname{tlng}=\mathrm{es}$.

Malengreau, J., y Albeti - Mayer. (1974). Comuneros y "Empresarios" en el intercambio. En Malengreau, J. Instituto de estudios Peruanos. Reciprocidad e intercambio en los andes del Perú, Pp. 175-205. Lima, Perú: IEP ediciones.

Mayta, Y. (2019). La "Dulce misa" como ritual de prosperidad en el santuario de San Bartolomé de Juli. Puno: (Tesis de pregrado) Universidad Nacional del Altiplano.

Padilla, A. y Ciruela, AM. (2018) Mujeres empresarias indígenas, cultura y capital social. El caso de la comunidad quechua de Tiquipaya (Bolivia). Foro internacional de estudios de la mujer. 69, 159-170. 10-1016/j. wsif.2018.05.012

Reyes, JL., Garzón, MA., y Tapia, B. (2018). Diseño y validación de una escala tipo likert para establecer características emprendedoras. Dimensión Empresarial, 16(2), 135- 160. 10.15665/dem.v16i2.1599

Salas Obregón, F., Veliz Quispe, T., \& Veliz Gonzales, C. F. (2019). Reinvención de la cultura en la actividad turística del mundo andino quechua, caso Urubamba - cusco, 2018. Revista De Investigaciones De La Escuela De Posgrado De La UNA PUNO, 8(3), 1129 - 1138. 10.26788/epg.v8i3.960

Santos, A. (2007). Ser Aymara en Lima. Diario cabildo abierto,Puno reportaje setiembre 2007, Pág. 10

Arnold, S. P. (2004). Ritualidad y cambios el caso Aymara. Puno, Perú: Instituto de estudios Aymaras (Idea) 
Tahmasebi, A. y Askaribezayeh, F. (2021) Microfinanzas y formación del capital social: Un enfoque de análisis de redes sociales. Ciencia de la planificación económica. Volumen 76,2021, 100978. 10.1016/j. seps.2020.100978
Umaña, M. (2019). Capital semilla y la ecuación de inversión en emprendimiento. Anuario de Investigación: Universidad Católica de El Salvador. 8. 59-71. 10.5377/aiunicaes. v8i0.8341 